\title{
RELATIONS BETWEEN BANACH FUNCTION ALGEBRAS AND THEIR UNIFORM CLOSURES
}

\author{
TAHER G. HONARY
}

(Communicated by John B. Conway)

\begin{abstract}
Let $A$ be a Banach function algebra on a compact Hausdorff space $X$. In this paper we consider some relations between the maximal ideal space, the Shilov boundary and the Choquet boundary of $A$ and its uniform closure $\bar{A}$. As an application we determine the maximal ideal space, the Shilov boundary and the Choquet boundary of algebras of infinitely differentiable functions which were introduced by Dales and Davie in 1973.
\end{abstract}

For some notations, definitions, elementary and known results, one can refer to [2] and [3].

Let $X$ be a compact Hausdorff space and let $C(X)$ denote the space of all continuous complex valued functions on $X$. A function algebra on $X$ is a subalgebra of $C(X)$ which contains the constants and separates the points of $X$. If there is an algebra norm on $A$ so that $\|f \cdot g\| \leq\|f\| \cdot\|g\|$ for all $f, g \in A$, then $A$ is called a normed function algebra. A complete normed function algebra on $X$ is called a Banach function algebra on $X$. If the norm of a Banach function algebra is the uniform norm on $X$; i.e. $\|f\|_{X}=\sup _{x \in X}|f(x)|$, it is called a uniform algebra on $X$. If $A$ is a function algebra on $X$, then $\bar{A}$, the uniform closure of $A$, is a uniform algebra on $X$.

If $(A,\|\cdot\|)$ is a Banach function algebra on $X$, for every $x \in X$ the map $\phi_{x}: A \rightarrow \mathbf{C}$, defined by $\phi_{x}(f)=f(x)$, is a nonzero continuous complex homomorphism on $A$ and so $\phi_{x} \in M_{A}$, where $M_{A}$ is the maximal ideal space of $A$. We call $\phi_{x}$ the evaluation homomorphism at $x$. Clearly for every $f \in A$,

$$
\|f\|_{X}=\sup _{x \in X}|f(x)|=\sup _{x \in X}\left|\phi_{x}(f)\right| \leq \sup _{\phi \in M_{A}}|\phi(f)|=\|\hat{f}\|_{M_{A}} \leq\|f\|,
$$

where $\hat{f}$ is the Gelfand transform of $f$.

The Banach function algebra $A$ on $X$ is called natural, if every $\phi \in M_{A}$ is given by an evaluation homomorphism $\phi_{x}$ at some $x \in X$; or, in other

Received by the editors February 9, 1989.

1980 Mathematics Subject Classification (1985 Revision). Primary 46J10; Secondary 46J20.

Key words and phrases. Banach function algebras, uniform algebras, maximal ideal space, Shilov boundary, Banach algebras of differentiable functions. 
words, the map $x \rightarrow \phi_{x}$ is surjective. In fact $M_{A}$ and $X$ are homeomorphic through this mapping whenever $A$ is natural. When $A$ is natural we usually write $M_{A} \cong X$.

The Shilov boundary of $A$ (with respect to $X$ ) is the smallest closed boundary of $A$ (with respect to $X$ ) and it is denoted by $\Gamma(A, X)$.

If $A$ is a function algebra on $X$ and $A^{*}$ is the conjugate (dual) space of $\left(A,\|\cdot\|_{X}\right)$, then the state space of $A$, denoted by $K(A)$, is defined by $K(A)=$ $\left\{\phi \in A^{*}:\|\phi\|=\phi(1)=1\right\} . K(A)$ is a weak ${ }^{*}$-compact Hausdorff convex subset of the closed unit ball in $A^{*}$. The Choquet boundary of $A$ (with respect to $X)$ is the set of all $x \in X$ for which $\phi_{x}$ is an extreme point of $K(A)$ and it is denoted by $\operatorname{Ch}(A, X)$. It is known that $\overline{\operatorname{Ch}}(A, X)=\Gamma(A, X)$.

Now we require a number of definitions and results which we now state briefly. For further details one can refer to [1].

Let $X$ be a perfect compact plane set. A complex-valued function $f$ on $X$ is differentiable at $z_{0}$ if

$$
f^{\prime}\left(z_{0}\right)=\lim _{\substack{z \rightarrow z_{0} \\(z \in X)}} \frac{f(z)-f\left(z_{0}\right)}{z-z_{0}}
$$

exists.

The algebra of functions on $X$ with continuous $k$ th derivative is denoted by $D^{k}(X) . D^{\infty}(X)$ is the algebra of functions with derivatives of all orders. Let $\left\{M_{k}\right\}_{k=0}^{\infty}$ be a sequence of positive numbers such that $M_{0}=1$ and for every $k \geq 1$,

$$
\frac{M_{k}}{M_{r} \cdot M_{k-r}} \geq\left(\begin{array}{l}
k \\
r
\end{array}\right) \quad(r=0,1, \ldots, k) .
$$

We define

$$
D\left(X,\left\{M_{k}\right\}\right)=\left\{f \in D^{\infty}(X): \sum_{k=0}^{\infty} \frac{\left\|f^{(k)}\right\|_{X}}{M_{k}}<\infty\right\}
$$

and a norm on $D\left(X,\left\{M_{k}\right\}\right)$ by

$$
\|f\|_{D}=\sum_{k=0}^{\infty} \frac{\left\|f^{(k)}\right\|_{X}}{M_{k}} .
$$

Taking $M_{r}=r !(r=0,1, \ldots, k)$ and $1 / M_{r}=0(r=k+1, \ldots)$, we regard $D^{k}(X)$ as being an algebra of the type $D\left(X,\left\{M_{k}\right\}\right)$. Hence for $f \in D^{k}$,

$$
\|f\|_{D^{k}}=\sum_{r=0}^{k} \frac{\left\|f^{(r)}\right\|_{X}}{r !}
$$

Clearly $\left(D^{k}(X),\|\cdot\|_{D^{k}}\right)$ and $\left(D\left(X,\left\{M_{k}\right\}\right),\|\cdot\|_{D}\right)$ are normed function algebras on $X$.

A compact plane set $X$ is called uniformly regular if it is rectifiably arcwise connected and if the geodesic metric is uniformly equivalent to the Euclidean 
metric on $X$. In other words, there exists a constant $C$ such that for every $z, w \in X, \delta(z, w) \leq C|z-w|$, where $\delta(z, w)$ is the geodesic distance between $z$ and $w$. When $X$ is uniformly regular $D^{k}(X)$ and $D\left(X,\left\{M_{k}\right\}\right)$ are Banach function algebras on $X$ with the appropriate norm [1]. If $D$ is any of the preceding algebras then the subalgebra of $D$ which is generated by the coordinate function $z$ is denoted by $D_{P}$ and the subalgebra generated by the rational functions with poles off $X$ that belong to $D$ is denoted by $D_{R}$. Clearly $D_{P}$ and $D_{R}$ are Banach function algebras on $X$ and $D_{P} \subseteq D_{R} \subseteq D \subseteq D^{k}(X)$ for each $k(k=1,2, \ldots)$. Moreover, according to Lemma (1.5) in [1], $D^{1}(X) \subseteq R(X)$, where $R(X)$ is the uniform closure of rational functions on the uniformly regular set $X$ with poles off $X$.

Let $P_{0}=1$ and $P_{k}=\sqrt[k]{M_{k} / k !}$ for $k \geq 1$ (we take $P_{k}=\infty$ if $M_{k}=\infty$ ). It is easy to see that if $P_{k}$ is bounded, none of the preceding algebras are natural for any $X$. We are thus interested in the case when $P_{k}$ is unbounded. We include the algebra $D^{k}(X)$ under this head. It is easy to show that $D_{R}\left(X,\left\{M_{k}\right\}\right)$ is natural when $P_{k} \rightarrow \infty$ as $k \rightarrow \infty$ (see [1]).

It was also proved in [1] that $M_{D_{P}} \cong \widehat{X}$, where $\widehat{X}$ is the polynomial convex hull of $X$. We shall give another proof for this result which is much simpler than that in [1].

In this paper $X$ always stands for a compact Hausdorff space. If $A$ is a function algebra on $X$ then it is easy to see that the restriction map $F: K(\bar{A}) \rightarrow$ $K(A)$ defined by $F(\psi)=\psi \mid A$ for $\psi \in K(\bar{A})$ is a homomorphism and so $K(\bar{A}) \cong K(A)$. Hence, ex $K(A) \cong \operatorname{ex} K(\bar{A})$ where ex $K(A)$ is the set of extreme points of $K(A)$. Therefore $\operatorname{Ch}(A, X)=\operatorname{Ch}(\bar{A}, X)$. Since $\overline{\mathrm{Ch}}(A, X)=$ $\Gamma(A, X)$ and $\overline{\operatorname{Ch}}(\bar{A}, X)=\Gamma(\bar{A}, X)$ we also have $\Gamma(A, X)=\Gamma(\bar{A}, X)$.

Theorem. If $A$ is a Banach function algebra on $X$ then the restriction map $F: M_{\bar{A}} \rightarrow M_{A}$ is a homomorphism if and only if $\|\hat{f}\|=\|f\|_{X}$ for every $f \in A$.

Remark. We usually write $M_{\bar{A}} \cong M_{A}$ whenever the above restriction map is a homeomorphism.

Proof. Let $F: M_{\bar{A}} \rightarrow M_{A}$ be the restriction map defined by $F(\psi)=\left.\psi\right|_{A}$. Clearly $\phi=\left.\psi\right|_{A}$ is a nonzero complex homomorphism on $A$ and so $F(\psi)=$ $\phi \in M_{A}$ for every $\psi \in M_{\bar{A}}$. If $f \in \bar{A}$ and $\phi \in M_{A}$ there exists a sequence $\left\{f_{n}\right\}$ in $A$ such that $\left\|f_{n}-f\right\|_{X} \underset{n \rightarrow \infty}{\longrightarrow} 0$ and so by the hypothesis $\left|\phi\left(f_{n}\right)-\phi\left(f_{m}\right)\right| \leq$ $\left\|f_{n}-f_{m}\right\|_{X} \underset{n, m \rightarrow \infty}{\longrightarrow} 0$. Hence, $\lim _{n \rightarrow \infty} \phi\left(f_{n}\right)$ exists and we can define $\psi$ on $\bar{A}$ by $\psi(f)=\lim _{n \rightarrow \infty} \phi\left(f_{n}\right)$. It is easy to see that $\psi \in M_{\bar{A}}$ and in fact $\psi$ is the extension of $\phi$ to $\bar{A}$, i.e. $F(\psi)=\phi$. Therefore $F$ is one-one and onto. Considering the weak ${ }^{*}$-topology of $M_{A}$ and $M_{\bar{A}}$, it is easy to show that $F$ and $F^{-1}$ are continuous. Hence $F$ is a homomorphism and so $M_{A} \cong M_{\bar{A}}$.

Conversely let the restriction map $F: M_{\bar{A}} \rightarrow M_{A}$ be a homomorphism. If $\phi \in M_{A}$ and $f \in A$, then there exists $\psi \in M_{\bar{A}}$ such that $\phi=F(\psi)=\psi \mid A$ and 
so $|\phi(f)|=|\psi(f)| \leq\|f\|_{X}$. Hence for every $f \in A,\|\hat{f}\|_{M_{A}}=\sup _{\phi \in M_{A}}|\phi(f)| \leq$ $\|f\|_{X}$.

Note that if $A$ is a natural Banach function algebra on $X$, then $\bar{A}$ is also natural (uniform algebra) on $X$, but the converse is not true.

Corollary 1. If $A$ and $B$ are Banach function algebras on $X$ such that

(i) $A \subseteq B$,

(ii) there exists $K>0$ such that for every $f \in A,\|f\|_{A} \leq K\|f\|_{B}$,

(iii) $B$ is natural, then $M_{A} \cong M_{\bar{A}}$. In particular if $\bar{A}=\bar{B}$ then $A$ is also natural.

Note that with the above hypotheses $A$ is in fact a closed subalgebra of $B$ under the norm of $B$.

Proof. By the spectral radius theorem and the above hypotheses for every $f \in A$.

$$
\|\hat{f}\|_{M_{A}}=\lim _{n \rightarrow \infty}\left\|f^{n}\right\|_{A}^{1 / n} \leq \lim _{n \rightarrow \infty} K^{1 / n}\left\|f^{n}\right\|_{B}^{1 / n}=\|\hat{f}\|_{M_{B}}=\|f\|_{X} .
$$

Hence by the theorem $M_{A} \cong M_{\bar{A}}$. Since $B$ is natural $\bar{B}$ is also natural. Thus if $\bar{A}=\bar{B}$ then $\bar{A}$ is also natural and so $M_{A} \cong X$, i.e. $A$ is also natural.

The converse of Corollary 1 is not true, in the following sense: If $A$ and $B$ are Banach function algebras on $X$ with the properties (i), (ii), $\bar{A}=\bar{B}$ and $A$ is natural, then $B$ may not be natural as the following example shows.

Example. Let

$$
h(x)=\sum_{n=1}^{\infty} 2^{-n} \sin \left(2^{n^{2}} \pi x\right) .
$$

Clearly $h$ is continuous and $|h(x)|<1$ on $\mathbf{R}$ and it is possible to prove that for every real $x$ and positive integer $n$ there exists $t \in\left[x, x+2^{-n^{2}}\right]$ such that $|h(t)-h(x)|>2^{-n-2}$.

Let $X=[0,1], A=D^{1}(X)$ and

$$
B=\left\{f: f=\sum_{n=0}^{\infty} f_{n} \cdot h^{n}, f_{n} \in A, \sum_{n=0}^{\infty}\left\|f_{n}\right\|_{D^{1}}<\infty\right\} .
$$

Clearly $A \subseteq B$ and $B$ is a function algebra on $X$. In order to define a norm on $B$ we first prove the above representation of elements of $B$ is unique.

Suppose $\sum_{n=0}^{\infty} f_{n} \cdot h^{n}=0$ on $X$ and $\sum_{n=0}^{\infty}\left\|f_{n}\right\|_{D^{1}} \leq 1$. To prove $f_{n}=0$ on $X$ for all $n \geq 0$, let $0<x<1$ and $0<\varepsilon$ be small enough such that $\varepsilon+x<1$. Since $f_{n} \in D^{1}(X)$ there exists $0<\theta<1$ such that $f_{n}(\varepsilon+x)-f_{n}(x)=\varepsilon f_{n}^{\prime}(\theta)$. Therefore

$$
\begin{aligned}
\left|\sum_{n=0}^{\infty} f_{n}(x) h^{n}(\varepsilon+x)\right| & =\left|\sum_{n=0}^{\infty}\left(f_{n}(x)-f_{n}(\varepsilon+x)\right) h^{n}(\varepsilon+x)\right| \\
& \leq \sum_{n=0}^{\infty} \varepsilon\left\|f_{n}^{\prime}\right\|_{X}<\varepsilon \sum_{n=0}^{\infty}\left\|f_{n}\right\|_{D^{1}} \leq \varepsilon .
\end{aligned}
$$


Now we define the sequence $\left\{a_{n}\right\}$ by

$$
\sum_{n=0}^{\infty} f_{n}(x)[h(x)+t]^{n}=\sum_{n=0}^{\infty} a_{n} t^{n}
$$

for small enough $|t|$. If the $a_{n}$ are not all zero and $m=\min \left\{n: a_{n} \neq 0\right\}$, then for small enough $\varepsilon$, writing $h(\varepsilon+x)-h(x)=t$, we have

$$
\left|\sum_{n=0}^{\infty} f_{n}(x) h^{n}(\varepsilon+x)\right|=\left|\sum_{n=m}^{\infty} a_{n} t^{n}\right|>\frac{1}{2}\left|a_{m}\right||t|^{m} .
$$

Therefore for small enough $\varepsilon>0, \frac{1}{2}\left|a_{m}\right||t|^{m}=\frac{1}{2}\left|a_{m}\right||h(\varepsilon+x)-h(x)|^{m}<\varepsilon$, which contradicts the property of $h$. Hence, $a_{n}=0$ and so $f_{n}(x)=0$ for all $n \geq 0$. Since $x$ is arbitrary, $f_{n}=0$ on $X$ for all $n \geq 0$.

Now we can define a norm on $B$ by $\|f\|_{B}=\sum_{n=0}^{\infty}\left\|f_{n}\right\|_{D^{1}}(f \in B)$. It is easy to show that $\left(B,\|\cdot\|_{B}\right)$ is a Banach function algebra on $X$. Since $A$ is selfadjoint by the Stone-Weierstrass theorem $\bar{A}=C(X)$ and so $\bar{A}=\bar{B}=C(X)$. Moreover for every $f \in A,\|f\|_{A}=\|f\|_{B}$. Although $A$ and $\bar{B}$ are natural, $B$ is not natural. Because for every $x \in X$ and every (non-real) complex number $\lambda$ in the closed unit disk we can define an element $\phi_{x, \lambda}$ of $M_{B}$ by

$$
\phi_{x, \lambda}\left(\sum_{n=0}^{\infty} f_{n} \cdot h^{n}\right)=\sum_{n=0}^{\infty} f_{n}(x) \lambda^{n} .
$$

It is easy to see that $\phi_{x, \lambda} \in M_{B}$ but it is not an evaluation homomorphism on $X$. Note that $h \in B$ but $h \notin D^{1}(X)$.

Now let $X$ be a uniformly regular compact plane set and the sequence $\left\{M_{k}\right\}$ satisfy condition $(*)$. It is easily seen that $\bar{D}_{P}\left(X,\left\{M_{k}\right\}\right)=P(X)$. If $P_{k} \rightarrow \infty$ as $k \rightarrow \infty$ then every rational function with poles off $X$ belongs to $D_{R}\left(X,\left\{M_{k}\right\}\right)$ and so $\bar{D}_{R}\left(X,\left\{M_{k}\right\}\right)=\bar{D}\left(X,\left\{M_{k}\right\}\right)=R(X)$. Also it follows that:

(i) $\operatorname{Ch}\left(D_{P}, X\right)=\operatorname{Ch}(P(X), X) \subseteq \partial \widehat{X}$,

(ii) $\Gamma\left(D_{P}, X\right)=\Gamma(P(X), X)=\partial \widehat{X}$,

(iii) $\operatorname{Ch}\left(D_{R}, X\right)=\mathrm{Ch}(D, X)=\mathrm{Ch}(R(X), X) \subseteq \partial X$,

(iv) $\Gamma\left(D_{R}, X\right)=\Gamma(D, X)=\Gamma(R(X), X)=\partial X$,

where $\partial X$ is the topological boundary of $X$.

As an application of Corollary 1, we give another proof of a theorem of Dales and Davie [1].

Corollary 2. Let $X$ be a uniformly regular compact plane set and the sequence $\left\{M_{k}\right\}$ satisfy condition $(*)$. If $P_{k} \rightarrow \infty$ as $k \rightarrow \infty$ then $M_{D_{P}} \cong \widehat{X}$.

Proof. Clearly $D_{P} \subseteq D_{R}$ and for every $f \in D_{P},\|f\|_{D_{P}}=\|f\|_{D_{R}}$. Since $D_{R}$ is natural, by Corollary $1, M_{\bar{D}_{P}} \cong M_{D_{P}}$. But $\bar{D}_{P}=P(X)$ and $M_{P(X)} \cong \widehat{X}$. So we have $M_{D_{P}} \cong \widehat{X}$. 


\section{ACKNOWLEDGMENT}

Special thanks are due to the University For Teacher Education in Tehran who supported me financially throughout this work.

\section{REFERENCES}

1. H. G. Dales and A. M. Davie, Quasianalytic Banach function algebras, J. Funct. Anal. (1) 13 (1973), 28-50.

2. T. W. Gamelin, Uniform algebras, Prentice-Hall, Englewood Cliffs, NJ, 1969.

3. C. E. Rickart, General theory of Banach algebras, Van Nostrand, Princeton, NJ, 1960.

Department of Mathematics, University for Teacher Education, Tehran 15614, I. R. IRAN 